\title{
Author Correction: CHD3 helicase domain mutations cause a neurodevelopmental syndrome with macrocephaly and impaired speech and language
}

Lot Snijders Blok, Justine Rousseau, Joanna Twist et al. ${ }^{\#}$

Correction to: Nature Communications https://doi.org/10.1038/s41467-018-06014-6, published online 05 November 2018.

The HTML and PDF versions of this Article were updated after publication to remove images of one individual from Fig. 1.

Published online: 02 May 2019

\begin{abstract}
(c) Open Access This article is licensed under a Creative Commons Attribution 4.0 International License, which permits use, sharing, adaptation, distribution and Ceproduction in any medium or format, as long as you give appropriate credit to the original author(s) and the source, provide a link to the Creative Commons license, and indicate if changes were made. The images or other third party material in this article are included in the article's Creative Commons license, unless indicated otherwise in a credit line to the material. If material is not included in the article's Creative Commons license and your intended use is not permitted by statutory regulation or exceeds the permitted use, you will need to obtain permission directly from the copyright holder. To view a copy of this license, visit http://creativecommons.org/licenses/by/4.0/.
\end{abstract}

(c) The Author(s) 2019

Lot Snijders Blok (1) ${ }^{1,2,3}$, Justine Rousseau ${ }^{4}$, Joanna Twist ${ }^{5}$, Sophie Ehresmann ${ }^{4}$, Motoki Takaku ${ }^{5}$, Hanka Venselaar ${ }^{6}$, Lance H. Rodan ${ }^{7}$, Catherine B. Nowak ${ }^{7}$, Jessica Douglas ${ }^{7}$, Kathryn J. Swoboda ${ }^{8}$, Marcie A. Steeves ${ }^{9}$, Inderneel Sahai ${ }^{9}$, Connie T.R.M. Stumpe ${ }^{10}$, Alexander P.A. Stegmann ${ }^{10}$, Patricia Wheeler ${ }^{11}$, Marcia Willing ${ }^{12}$, Elise Fiala ${ }^{12}$, Aaina Kochhar ${ }^{13}$, William T. Gibson ${ }^{14,15}$, Ana S.A. Cohen (1) ${ }^{14,15}$, Ruky Agbahovbe ${ }^{14,15}$, A. Micheil Innes (10 ${ }^{16}$, P.Y.Billie Au ${ }^{16}$, Julia Rankin ${ }^{17}$, Ilse J. Anderson ${ }^{18}$, Steven A. Skinner ${ }^{19}$, Raymond J. Louie ${ }^{19}$, Hannah E. Warren ${ }^{19}$, Alexandra Afenjar ${ }^{20}$, Boris Keren ${ }^{21,22}$, Caroline Nava ${ }^{21,22,23}$, Julien Buratti ${ }^{21}$, Arnaud Isapof ${ }^{24}$, Diana Rodriguez ${ }^{25}$, Raymond Lewandowski ${ }^{26}$, Jennifer Propst ${ }^{26}$, Ton van Essen ${ }^{27}$, Murim Choi ${ }^{28}$, Sangmoon Lee ${ }^{28}$, Jong H. Chae ${ }^{29}$, Susan Price ${ }^{30}$, Rhonda E. Schnur ${ }^{31}$, Ganka Douglas ${ }^{31}$, Ingrid M. Wentzensen ${ }^{31}$, Christiane Zweier ${ }^{32}$, André Reis ${ }^{32}$, Martin G. Bialer ${ }^{33}$, Christine Moore ${ }^{33}$, Marije Koopmans ${ }^{34}$, Eva H. Brilstra ${ }^{34}$, Glen R. Monroe ${ }^{34}$, Koen L.I. van Gassen ${ }^{34}$, Ellen van Binsbergen ${ }^{34}$, Ruth Newbury-Ecob ${ }^{35}$, Lucy Bownass ${ }^{35}$, Ingrid Bader ${ }^{36}$, Johannes A. Mayr ${ }^{37}$, Saskia B. Wortmann ${ }^{37,38,39}$, Kathy J. Jakielski ${ }^{40}$, Edythe A. Strand ${ }^{41}$, Katja Kloth $^{42}$, 
Tatjana Bierhals ${ }^{42}$, The DDD study, John D. Roberts ${ }^{5}$, Robert M. Petrovich ${ }^{5}$, Shinichi Machida ${ }^{43}$, Hitoshi Kurumizaka (1) ${ }^{43}$, Stefan Lelieveld ${ }^{1}$, Rolph Pfundt ${ }^{1}$, Sandra Jansen ${ }^{1,3}$, Pelagia Deriziotis ${ }^{2}$, Laurence Faivre ${ }^{44,45}$, Julien Thevenon ${ }^{44,45}$, Mirna Assoum ${ }^{44,45}$, Lawrence Shriberg ${ }^{46}$, Tjitske Kleefstra ${ }^{1,3}$, Han G. Brunner ${ }^{1,3,10}$, Paul A. Wade (10 ${ }^{5}$, Simon E. Fisher (10 ${ }^{2,3}$ \& Philippe M. Campeau (iD ${ }^{4,47}$

'Department of Human Genetics, Radboud University Medical Center, Nijmegen 6500HB, The Netherlands. ${ }^{2}$ Language and Genetics Department, Max Planck Institute for Psycholinguistics, Nijmegen 6500AH, The Netherlands. ${ }^{3}$ Donders Institute for Brain, Cognition and Behaviour, Radboud University, Nijmegen $6500 \mathrm{HE}$, The Netherlands. ${ }^{4} \mathrm{CHU}$ Sainte-Justine Research Center, Montreal QC H3T 1C5, Canada. ${ }^{5}$ National Institute of Environmental Health Sciences, Research Triangle Park NC 27709, USA. ${ }^{6}$ Centre for Molecular and Biomolecular Informatics, Radboud Institute for Molecular Life Sciences, Radboud University Medical Center, Nijmegen 6500HB, The Netherlands. 7 Division of Genetics and Genomics, Boston Children's Hospital, Boston MA 02115, USA. ${ }^{8}$ Department of Neurology, Massachusetts General Hospital and Harvard Medical School, Boston MA 02114, USA. ${ }^{9}$ Department of Medical Genetics, Massachusetts General Hospital, Boston MA 02114, USA. ${ }^{10}$ Department of Clinical Genetics and GROW-School for Oncology and Developmental Biology, Maastricht University Medical Center, Maastricht 6202AZ, The Netherlands. ${ }^{11}$ Nemours Childrens Clinic, Orlando FL 32827, USA. ${ }^{12}$ Division of Genetics and Genomic Medicine, Department of Pediatrics, Washington University School of Medicine, St. Louis MO 63110, USA. ${ }^{13}$ Valley Children's Hospital, Madera CA 93636, USA. ${ }^{14}$ British Columbia Children's Hospital Research Institute, Vancouver BC V5Z 4H4, Canada. ${ }^{15}$ Department of Medical Genetics, University of British Columbia, Vancouver BC V6H 3N1, Canada. ${ }^{16}$ Department of Medical Genetics and Alberta Children's Hospital Research Institute, Cumming School of Medicine, University of Calgary, Calgary AB T2N 4N1, Canada. ${ }^{17}$ Department of Clinical Genetics, Royal Devon and Exeter NHS Foundation Trust (Heavitree), Exeter EX2 5DW, UK. ${ }^{18}$ Division of Genetics, Department of Medicine, University of Tennessee Medical Center, Knoxville TN 37920, USA. ${ }^{19}$ Greenwood Genetic Center, Greenwood SC 29646, USA. ${ }^{20}$ GRC ConCer-LD, Sorbonne Universités, UPMC Univ Paris; Department of Medical Genetics and Centre de Référence Malformations et maladies congénitales du cervelet et déficiences intellectuelles de causes rares, Armand Trousseau Hospital, GHUEP, AP-HP, Paris 75012, France. ${ }^{21}$ AP-HP, Hôpital de la Pitié-Salpêtrière, Département de Génétique, Paris 75013, France. ${ }^{22}$ Groupe de Recherche Clinique (GRC) 'déficience intellectuelle et autisme' UPMC, Paris 75005, France. ${ }^{23}$ INSERM, U 1127, CNRS UMR 7225, Institut du Cerveau et de la Moelle épinière, ICM, Sorbonne Universités, UPMC Univ Paris 06 UMR S 1127, 75013 Paris, France. ${ }^{24}$ GRC ConCer-LD, Sorbonne Universités, UPMC Univ Paris 06; Department Child Neurology and Reference Center for Neuromuscular Diseases "Nord/Est/Ile-de-France", FILNEMUS, Armand Trousseau Hospital, GHUEP, AP-HP, Paris 75012, France. ${ }^{25}$ GRC ConCer-LD, Sorbonne Universités, UPMC Univ Paris 06; Department of Child Neurology and National Reference Center for Neurogenetic Disorders, Armand Trousseau Hospital, GHUEP, AP-HP, INSERM U1141, 75012 Paris, France. ${ }^{26}$ Clinical Genetics Division, Virginia Commonwealth University Health System, Richmond VA 23298, USA. ${ }^{27}$ Clinical Genetics Department, University Medical Center Groningen, Groningen 9700RB, The Netherlands. ${ }^{28}$ Department of Biomedical Sciences, Seoul National University College of Medicine, Seoul 08826, Republic of Korea. ${ }^{29}$ Department of Pediatrics, Seoul National University College of Medicine, Seoul National University Children's Hospital, Seoul 08826, Republic of Korea. ${ }^{30}$ Oxford University Hospitals NHS Foundation Trust, Oxford OX3 7HE, UK. ${ }^{31}$ GeneDx, Gaithersburg MD 20877, USA. ${ }^{32}$ Institute of Human Genetics, Friedrich-Alexander-Universität Erlangen-Nürnberg, Erlangen 91054 , Germany. ${ }^{33}$ Northwell Health, Division of Medical Genetics and Genomics, Great Neck NY 11021, USA. ${ }^{34}$ Department of Genetics, University Medical Center Utrecht, Utrecht University, Utrecht 3508AB, The Netherlands. ${ }^{35}$ University Hospitals Bristol, Department of Clinical Genetics, St Michael's Hospital, Bristol BS2 8EG, UK. ${ }^{36}$ Department of Clinical Genetics, University Children's Hospital, Paracelsus Medical University, Salzburg A-5020, Austria. ${ }^{37}$ Department of Pediatrics, Salzburger Landeskliniken and Paracelsus Medical University, Salzburg A-5020, Austria. ${ }^{38}$ Institute of Human Genetics, Technische Universität München, Munich 81675, Germany. ${ }^{39}$ Institute of Human Genetics, Helmholtz Zentrum München, Neuherberg 85764, Germany. ${ }^{40}$ Communication Sciences and Disorders, Augustana College, Rock Island IL 61201, USA. ${ }^{41}$ Department of Neurology, Mayo Clinic, Rochester MN 55905, USA. ${ }^{42}$ Institute of Human Genetics, University Medical Center Hamburg-Eppendorf, Hamburg 20246, Germany. ${ }^{43}$ Waseda University, Tokyo 169-8050, Japan. ${ }^{44}$ Equipe Génétique des Anomalies du Développement, Université de BourgogneFranche Comté, Dijon 21070, France. ${ }^{45}$ Centre de Génétique et Centre de Référence Anomalies du Développement et Syndromes Malformatifs, FHU TRANSLAD, Hôpital d'Enfants, CHU Dijon et Université de Bourgogne, Dijon 21079, France. ${ }^{46}$ Waisman Center, Phonology Project, Madison WI 53705-2280, USA. ${ }^{47}$ Sainte-Justine Hospital, University of Montreal, Montreal QC H3T 1C5, Canada. These authors contributed equally: Lot Snijders Blok, Justine Rousseau, Joanna Twist. These authors jointly supervised this work: Simon E. Fisher, Philippe M. Campeau. A full list of consortium members appears below.

\section{The DDD study}

Jeremy F. McRae ${ }^{48}$, Stephen Clayton ${ }^{48}$, Tomas W. Fitzgerald ${ }^{48}$, Joanna Kaplanis ${ }^{48}$, Elena Prigmore ${ }^{48}$, Diana Rajan ${ }^{48}$, Alejandro Sifrim ${ }^{48}$, Stuart Aitken ${ }^{49}$, Nadia Akawi ${ }^{48}$, Mohsan Alvi $^{50}$, Kirsty Ambridge ${ }^{48}$, Daniel M. Barrett ${ }^{48}$, Tanya Bayzetinova ${ }^{48}$, Philip Jones ${ }^{48}$, Wendy D. Jones ${ }^{48}$, Daniel King ${ }^{48}$, Netravathi Krishnappa ${ }^{48}$, Laura E. Mason ${ }^{48}$, Tarjinder Singh ${ }^{48}$, Adrian R. Tivey ${ }^{48}$, Munaza Ahmed ${ }^{51,52,53}$, Uruj Anjum ${ }^{54}$, Hayley Archer ${ }^{55,56}$, Ruth Armstrong ${ }^{57}$, Jana Awada ${ }^{48}$, Meena Balasubramanian ${ }^{58}$, Siddharth Banka ${ }^{59}$, Diana Baralle $51,52,53$, Angela Barnicoat ${ }^{60}$, Paul Batstone ${ }^{61}$, David Baty ${ }^{62}$, Chris Bennett ${ }^{63}$, Jonathan Berg ${ }^{62}$, Birgitta Bernhard ${ }^{64}$, A. Paul Bevan ${ }^{48}$, Maria Bitner-Glindzicz ${ }^{60}$, Edward Blair ${ }^{65}$, Moira Blyth ${ }^{63}$, David Bohanna ${ }^{66}$, Louise Bourdon ${ }^{64}$, David Bourn ${ }^{67}$, Lisa Bradley ${ }^{68}$, Angela Brady ${ }^{64}$, Simon Brent ${ }^{48}$, Carole Brewer $^{69}$, Kate Brunstrom ${ }^{60}$, David J. Bunyan ${ }^{51,52,53}$, John Burn ${ }^{67}$, Natalie Canham ${ }^{64}$, Bruce Castle ${ }^{69}$, Kate Chandler ${ }^{59}$, Elena Chatzimichali ${ }^{48}$, Deirdre Cilliers ${ }^{65}$, Angus Clarke ${ }^{55,56}$, Susan Clasper ${ }^{65}$, Jill Clayton-Smith ${ }^{59}$, Virginia Clowes ${ }^{64}$, Andrea Coates ${ }^{63}$, Trevor Cole ${ }^{66}$, Irina Colgiu ${ }^{48}$, Amanda Collins ${ }^{51,52,53}$, 
Morag N. Collinson $51,52,53$, Fiona Connell ${ }^{70}$, Nicola Cooper ${ }^{66}$, Helen Cox ${ }^{66}$, Lara Cresswell ${ }^{71}$, Gareth Cross $^{72}$, Yanick Crow ${ }^{59}$, Mariella D'Alessandro ${ }^{61}$, Tabib Dabir ${ }^{68}$, Rosemarie Davidson ${ }^{73}$, Sally Davies ${ }^{55,56}$, Dylan de Vries ${ }^{48}$, John Dean ${ }^{61}$, Charu Deshpande ${ }^{70}$, Gemma Devlin ${ }^{69}$, Abhijit Dixit ${ }^{72}$, Angus Dobbie ${ }^{63}$, Alan Donaldson ${ }^{74}$, Dian Donnai ${ }^{59}$, Deirdre Donnelly ${ }^{68}$, Carina Donnelly ${ }^{59}$, Angela Douglas ${ }^{75}$, Sofia Douzgou ${ }^{59}$, Alexis Duncan ${ }^{73}$, Jacqueline Eason ${ }^{72}$, Sian Ellard ${ }^{69}$, Ian Ellis ${ }^{75}$, Frances Elmslie ${ }^{54}$, Karenza Evans ${ }^{55,56}$, Sarah Everest ${ }^{69}$, Tina Fendick ${ }^{70}$, Richard Fisher ${ }^{67}$, Frances Flinter ${ }^{70}$, Nicola Foulds ${ }^{51,52,53}$, Andrew Fry ${ }^{55,56}$, Alan Fryer ${ }^{75}$, Carol Gardiner ${ }^{73}$, Lorraine Gaunt ${ }^{59}$, Neeti Ghali ${ }^{64}$, Richard Gibbons ${ }^{65}$, Harinder Gill ${ }^{76}$, Judith Goodship ${ }^{67}$, David Goudie ${ }^{62}$, Emma Gray ${ }^{48}$, Andrew Green ${ }^{76}$, Philip Greene ${ }^{48}$, Lynn Greenhalgh ${ }^{75}$, Susan Gribble ${ }^{48}$, Rachel Harrison ${ }^{72}$, Lucy Harrison ${ }^{51,52,53}$, Victoria Harrison ${ }^{51,52,53}$, Rose Hawkins ${ }^{74}$, Liu He $e^{48}$, Stephen Hellens ${ }^{67}$, Alex Henderson ${ }^{67}$, Sarah Hewitt ${ }^{63}$, Lucy Hildyard ${ }^{48}$, Emma Hobson ${ }^{63}$, Simon Holden ${ }^{57}$, Muriel Holder ${ }^{64}$, Susan Holder ${ }^{64}$, Georgina Hollingsworth ${ }^{60}$, Tessa Homfray ${ }^{54}$, Mervyn Humphreys ${ }^{68}$, Jane Hurst ${ }^{60}$, Ben Hutton ${ }^{48}$, Stuart Ingram ${ }^{58}$, Melita Irving ${ }^{70}$, Lily Islam ${ }^{66}$, Andrew Jackson ${ }^{49}$, Joanna Jarvis ${ }^{66}$, Lucy Jenkins ${ }^{60}$, Diana Johnson ${ }^{58}$, Elizabeth Jones ${ }^{59}$, Dragana Josifova ${ }^{70}$, Shelagh Joss ${ }^{73}$, Beckie Kaemba ${ }^{71}$, Sandra Kazembe ${ }^{71}$, Rosemary Kelsell ${ }^{48}$, Bronwyn Kerr ${ }^{59}$, Helen Kingston ${ }^{59}$, Usha Kini ${ }^{65}$, Esther Kinning ${ }^{73}$, Gail Kirby ${ }^{66}$, Claire Kirk ${ }^{68}$, Emma Kivuva ${ }^{69}$, Alison Kraus ${ }^{63}$, Dhavendra Kumar ${ }^{55,56}$, V.K.Ajith Kumar ${ }^{60}$, Katherine Lachlan ${ }^{51,52,53}$, Wayne Lam ${ }^{49}$, Anne Lampe ${ }^{49}$, Caroline Langman ${ }^{70}$, Melissa Lees ${ }^{60}$, Derek Lim ${ }^{66}$, Cheryl Longman ${ }^{73}$, Gordon Lowther ${ }^{73}$, Sally A. Lynch ${ }^{76}$, Alex Magee ${ }^{68}$, Eddy Maher ${ }^{49}$, Alison Male ${ }^{60}$, Sahar Mansour ${ }^{54}$, Karen Marks ${ }^{54}$, Katherine Martin ${ }^{72}$, Una Maye ${ }^{75}$, Emma McCann ${ }^{77}$, Vivienne McConnell ${ }^{68}$, Meriel McEntagart ${ }^{54}$, Ruth McGowan ${ }^{61}$, Kirsten McKay ${ }^{66}$, Shane McKee ${ }^{68}$, Dominic J. McMullan ${ }^{66}$, Susan McNerlan ${ }^{68}$, Catherine McWilliam ${ }^{61}$, Sarju Mehta ${ }^{57}$, Kay Metcalfe ${ }^{59}$, Anna Middleton ${ }^{48}$, Zosia Miedzybrodzka ${ }^{61}$, Emma Miles ${ }^{59}$, Shehla Mohammed ${ }^{70}$, Tara Montgomery ${ }^{67}$, David Moore ${ }^{49}$, Sian Morgan ${ }^{55,56}$, Jenny Morton ${ }^{66}$, Hood Mugalaasi ${ }^{55,56}$, Victoria Murday ${ }^{73}$, Helen Murphy ${ }^{59}$, Swati Naik ${ }^{66}$, Andrea Nemeth ${ }^{65}$, Louise Nevitt ${ }^{58}$, Andrew Norman ${ }^{66}$, Rosie O'Shea ${ }^{76}$, Caroline Ogilvie ${ }^{70}$, Kai-Ren Ong ${ }^{66}$, Soo-Mi Park ${ }^{57}$, Michael J. Parker ${ }^{58}$, Chirag Patel ${ }^{66}$, Joan Paterson ${ }^{57}$, Stewart Payne ${ }^{64}$, Daniel Perrett ${ }^{48}$, Julie Phipps ${ }^{65}$, Daniela T. Pilz ${ }^{73}$, Martin Pollard ${ }^{48}$, Caroline Pottinger ${ }^{77}$, Joanna Poulton ${ }^{65}$, Norman Pratt ${ }^{62}$, Katrina Prescott ${ }^{63}$, Abigail Pridham ${ }^{65}$, Annie Procter ${ }^{55,56}$, Hellen Purnell ${ }^{65}$, Oliver Quarrell ${ }^{58}$, Nicola Ragge ${ }^{66}$, Raheleh Rahbari $^{48}$, Josh Randall ${ }^{48}$, Lucy Raymond ${ }^{57}$, Debbie Rice ${ }^{62}$, Leema Robert ${ }^{70}$, Eileen Roberts ${ }^{74}$, Jonathan Roberts ${ }^{57}$, Paul Roberts ${ }^{63}$, Gillian Roberts ${ }^{75}$, Alison Ross ${ }^{61}$, Elisabeth Rosser ${ }^{60}$, Anand Saggar ${ }^{54}$, Shalaka Samant ${ }^{61}$, Julian Sampson ${ }^{55,56}$, Richard Sandford ${ }^{57}$, Ajoy Sarkar ${ }^{72}$, Susann Schweiger ${ }^{62}$, Richard Scott ${ }^{60}$, Ingrid Scurr ${ }^{74}$, Ann Selby ${ }^{72}$, Anneke Seller ${ }^{65}$, Cheryl Sequeira ${ }^{64}$, Nora Shannon ${ }^{72}$, Saba Sharif ${ }^{66}$, Charles Shaw-Smith ${ }^{69}$, Emma Shearing ${ }^{58}$, Debbie Shears ${ }^{65}$, Eamonn Sheridan ${ }^{63}$, Ingrid Simonic ${ }^{57}$, Roldan Singzon ${ }^{64}$, Zara Skitt ${ }^{59}$, Audrey Smith ${ }^{63}$, Kath Smith ${ }^{58}$, Sarah Smithson ${ }^{74}$, Linda Sneddon ${ }^{67}$, Miranda Splitt ${ }^{67}$, Miranda Squires ${ }^{63}$, Fiona Stewart ${ }^{68}$, Helen Stewart ${ }^{65}$, Volker Straub ${ }^{67}$, Mohnish Suri ${ }^{72}$, Vivienne Sutton ${ }^{75}$, Ganesh Jawahar Swaminathan ${ }^{48}$, Elizabeth Sweeney ${ }^{75}$, Kate Tatton-Brown ${ }^{54}$, Cat Taylor ${ }^{5}$, Rohan Taylor ${ }^{54}$, Mark Tein ${ }^{66}$, I. Karen Temple ${ }^{51,52,53}$, Jenny Thomson ${ }^{63}$, Marc Tischkowitz ${ }^{57}$, Susan Tomkins ${ }^{74}$, Audrey Torokwa ${ }^{51,52,53}$, Becky Treacy ${ }^{57}$, Claire Turner ${ }^{69}$, Peter Turnpenny ${ }^{69}$, Carolyn Tysoe ${ }^{69}$, Anthony Vandersteen ${ }^{64}$, Vinod Varghese ${ }^{55,56}$, Pradeep Vasudevan ${ }^{71}$, Parthiban Vijayarangakannan ${ }^{48}$, Julie Vogt ${ }^{66}$, Emma Wakeling ${ }^{64}$, Sarah Wallwark ${ }^{57}$, Jonathon Waters ${ }^{60}$, Astrid Weber ${ }^{75}$, Diana Wellesley ${ }^{51,52,53}$, Margo Whiteford ${ }^{73}$, Sara Widaa ${ }^{48}$, Sarah Wilcox ${ }^{57}$, Emily Wilkinson ${ }^{48}$, Denise Williams ${ }^{66}$, Nicola Williams ${ }^{73}$, Louise Wilson $^{60}$, Geoff Woods ${ }^{57}$, Christopher Wragg ${ }^{74}$, Michael Wright ${ }^{67}$, Laura Yates ${ }^{67}$, Michael Yau ${ }^{70}$, Chris Nellåker ${ }^{78,79,80}$, Michael Parker ${ }^{81}$, Helen V. Firth ${ }^{48,57}$, Caroline F. Wright ${ }^{48}$, David R. FitzPatrick ${ }^{48,49}$, Jeffrey C. Barrett ${ }^{48} \&$ Matthew E. Hurles ${ }^{48}$ 
${ }^{48}$ Wellcome Trust Sanger Institute, Wellcome Trust Genome Campus, Hinxton, Cambridge CB10 1SA, UK. ${ }^{49}$ MRC Human Genetics Unit, MRC IGMM, University of Edinburgh, Western General Hospital, Edinburgh EH4 2XU, UK. ${ }^{50}$ Department of Engineering Science, University of Oxford, Parks Road, Oxford OX1 3PJ, UK. ${ }^{51}$ Wessex Clinical Genetics Service, University Hospital Southampton, Princess Anne Hospital, Coxford Road, Southampton SO16 5YA, UK. ${ }^{52}$ Wessex Regional Genetics Laboratory, Salisbury NHS Foundation Trust, Salisbury District Hospital, Odstock Road, Salisbury, Wiltshire SP2 8BJ, UK. ${ }^{53}$ Faculty of Medicine, University of Southampton, Building 85, Life Sciences Building, Highfield Campus, Southampton SO17 1BJ, UK. ${ }^{54}$ South West Thames Regional Genetics Centre, St George's Healthcare NHS Trust, St George's, University of London, Cranmer Terrace, London SW17 ORE, UK. ${ }^{55}$ Institute of Medical Genetics, University Hospital of Wales, Heath Park, Cardiff CF14 4XW, UK. ${ }^{56}$ Department of Clinical Genetics, Block 12, Glan Clwyd Hospital, Rhyl, Denbighshire LL18 5UJ, UK. ${ }^{57}$ East Anglian Medical Genetics Service, Box 134, Cambridge University Hospitals NHS Foundation Trust, Cambridge Biomedical Campus, Cambridge CB2 OQQ, UK. ${ }^{58}$ Sheffield Regional Genetics Services, Sheffield Children's NHS Trust, Western Bank, Sheffield S10 2TH, UK. ${ }^{59}$ Manchester Centre for Genomic Medicine, St Mary's Hospital, Central Manchester University Hospitals NHSFoundation Trust, Manchester Academic Health Science Centre, Manchester M13 9WL, UK. ${ }^{60}$ North East Thames Regional Genetics Service, Great Ormond Street Hospital for Children NHS Foundation Trust, Great Ormond Street Hospital, Great Ormond Street, London WC1N3JH, UK. ${ }^{61}$ North of Scotland Regional Genetics Service, NHS Grampian, Department of Medical Genetics Medical School, Foresterhill, Aberdeen AB25 2ZD, UK. ${ }^{62}$ East of Scotland Regional Genetics Service, Human Genetics Unit, Pathology Department, NHS Tayside, Ninewells Hospital, Dundee DD1 9SY, UK. ${ }^{63}$ Yorkshire Regional Genetics Service, Leeds Teaching Hospitals NHS Trust, Department of Clinical Genetics, Chapel Allerton Hospital, Chapeltown Road, Leeds LS7 4SA, UK. ${ }^{64}$ North West Thames Regional Genetics Centre, North West London Hospitals NHS Trust, The Kennedy Galton Centre, Northwick Park and St Mark's NHS Trust Watford Road, Harrow HA1 3UJ, UK. ${ }^{65}$ Oxford Regional Genetics Service, Oxford Radcliffe Hospitals NHS Trust, The Churchill Old Road, Oxford OX3 7LJ, UK. ${ }^{66}$ West Midlands Regional Genetics Service, Birmingham Women's NHS Foundation Trust, Birmingham Women's Hospital, Edgbaston, Birmingham B15 2TG, UK. ${ }^{67}$ Northern Genetics Service, Newcastle upon Tyne Hospitals NHS Foundation Trust, Institute of Human Genetics, International Centre for Life, Central Parkway, Newcastle upon Tyne NE1 3BZ, UK. ${ }^{68}$ Northern Ireland Regional Genetics Centre, Belfast Health and Social Care Trust, Belfast City Hospital, Lisburn Road, Belfast BT9 7AB, UK. ${ }^{69}$ Peninsula Clinical Genetics Service, Royal Devon and Exeter NHS Foundation Trust, Clinical Genetics Department, Royal Devon \& Exeter Hospital (Heavitree), Gladstone Road, Exeter EX1 2ED, UK. ${ }^{70}$ South East Thames Regional Genetics Centre, Guy's and St Thomas' NHS Foundation Trust, Guy's Hospital, Great Maze Pond, London SE1 9RT, UK. ${ }^{71}$ Leicestershire Genetics Centre, University Hospitals of Leicester NHS Trust, Leicester Royal Infirmary (NHS Trust), Leicester LE1 5WW, UK. ${ }^{72}$ Nottingham Regional Genetics Service, City Hospital Campus, Nottingham University Hospitals NHS Trust, The Gables, Hucknall Road, Nottingham NG5 1PB, UK. ${ }^{73}$ West of Scotland Regional Genetics Service, NHS Greater Glasgow and Clyde, Institute of Medical Genetics, Yorkhill Hospital, Glasgow G3 8SJ, UK. ${ }^{74}$ Bristol Genetics Service (Avon, Somerset, Gloucs and West Wilts), University Hospitals Bristol NHS Foundation Trust, St Michael's Hospital, St Michael's Hill, Bristol BS2 8DT, UK. ${ }^{75}$ Merseyside and Cheshire Genetics Service, Liverpool Women's NHS Foundation Trust, Department of Clinical Genetics, Royal Liverpool Children's Hospital Alder Hey, Eaton Road, Liverpool L12 2AP, UK. ${ }^{76}$ National Centre for Medical Genetics, Our Lady's Children's Hospital, Crumlin, Dublin 12, Ireland. ${ }^{77}$ Department of Clinical Genetics, Block 12, Glan Clwyd Hospital, Rhyl, Denbighshire LL18 5UJ Wales, UK. ${ }^{78}$ Nuffield Department of Obstetrics \& Gynaecology, University of Oxford, Level 3, Women's Centre, John Radcliffe Hospital, Oxford OX3 9DU, UK. ${ }^{79}$ Institute of Biomedical Engineering, Department of Engineering Science, University of Oxford, Old Road Campus Research Building, Oxford OX3 7DQ, UK. ${ }^{80}$ Big Data Institute, University of Oxford, Roosevelt drive, Oxford OX3 7LF, UK. ${ }^{81}$ The Ethox Centre, Nuffield Department of Population Health, University of Oxford, Old Road Campus, Oxford OX3 7LF, UK 